\title{
Convertirse en madre durante la adolescencia: transiciones en el rol materno*
}

\author{
Becoming a mother during adolescence: transitions in the maternal role \\ Se tornar mãe na adolescência: transições no papel materno
}

\author{
Claudia Maria Moreno ${ }^{\text {a }}$ \\ Universidad Pedagógica y Tecnológica de Colombia, \\ Colombia \\ claudiamaria.moreno@uptc.edu.co \\ ORCID: https://orcid.org/0000-0002-1463-1546 \\ Alejandra Peralta Cruz \\ Universidad Pedagógica y Tecnológica de Colombia, \\ Colombia \\ ORCID: https://orcid.org/0000-0002-3168-4359 \\ Natalia Velásquez Monroy \\ Universidad Pedagógica y Tecnológica de Colombia, \\ Colombia \\ ORCID: https://orcid.org/0000-0002-1366-8881
}

Recibido: 20 Junio 2019

Aceptado: 03 Marzo 2020

DOI: https://doi.org/10.11144/Javeriana.ie22.cmda

Publicado: 22 Julio 2020

\section{Resumen:}

El artículo pretende dar a conocer la experiencia en el desarrollo de una estrategia de enfermería que promueva el cuidado integral de la madre adolescente, hijo, pareja o acompañante para la activación del rol materno durante el posparto, como parte del programa Creciendo Juntos de la ESE Hospital Regional de Duitama (Boyacá, Colombia). La estrategia de cuidado se diseñó a partir de la aplicación de la teoría "convertirse en madre", de Ramona Mercer, desde el sistema conceptual teórico-empírico y el modelo del marco lógico, así como desde el apoyo social, desarrollado en cuatro intervenciones: una visita domiciliaria inicial, seguimiento telefónico, grupo de madres y acompañantes en posparto y una segunda visita domiciliaria. De igual forma, los resultados se presentan en indicadores empíricos del desarrollo de la estrategia de cuidado en un grupo de 25 madres, cuyas intervenciones institucionales de salud se orientaron hacia la práctica del cuidado, como parte de la visibilidad asistencial de enfermería en madres adolescentes, y hacia la promoción de la maternidad desde la activación y normalización del rol en el proceso de convertirse en madre.

Palabras clave: periodo posparto, salud del adolescente, atención de enfermería.

\section{Abstract:}

The article aims to disclose the development experience of a nursing strategy that promotes comprehensive care of the adolescent mother, child, partner or companion for the activation of the maternal role during the postpartum period, as part of the Growing Together program of the ESE Duitama Regional Hospital (Boyaca, Colombia). The care strategy was designed based on the application of Ramona Mercer's "become a mother" theory, from a theoretical-empirical conceptual system and a logical framework model as well as from social support and was developed in four interventions: an initial home visit, phone call follow-up, group of mothers and companions in the postpartum period, and a second home visit. Similarly, results are presented by means of empirical indicators of the development of the care strategy in a group of 25 mothers for whom institutional health interventions were oriented towards the practice of care as part of the visibility of nursing care for adolescent mothers, and towards the promotion of motherhood from the activation and normalization of the role in the process of becoming a mother.

Keywords: postpartum period, adolescent health, nursing care.

\section{Resumo:}

O artigo visa divulgar a experiência no desenvolvimento de uma estratégia de enfermagem que promova o cuidado integral da mãe adolescente, criança, parceiro ou companheiro para a ativação do papel materno no pós-parto, como parte do programa Crescendo Juntos da ESE Hospital Regional de Duitama (Boyacá, Colômbia). A estratégia de cuidado foi elaborada a partir da

Notas de autor

a Autora de correspondencia. Correo electrónico: claudiamaria.moreno@uptc.edu.co 
aplicação da teoria "se tornar mãe", de Ramona Mercer, desde o sistema conceitual teórico-empírico e o modelo de Marco Lógico, bem como desde o apoio social, desenvolvido em quatro intervenções: uma visita domiciliária inicial, monitoramento telefónico, grupo de mães e acompanhantes em pós-parto e segunda visita domiciliária. De igual forma, os resultados são apresentados em indicadores empíricos de desenvolvimento da estratégia de cuidado em um grupo de 25 mães, cujas intervenções institucionais de saúde orientaram-se para a prática de cuidado, como parte da visibilidade assistencial de enfermagem em mães adolescentes, e para a promoção da maternidade desde a ativação e normalização do papel no processo de se tornar mãe.

Palavras-chave: período pós-parto, saúde do adolescente, atenção de enfermagem.

\section{Introducción}

Desde el inicio de la gestación, la madre adolescente transita por transformaciones emocionales, fisiológicas, anatómicas y socioculturales, y durante el periodo posparto se espera que se adapte para asumir el rol materno desde el cuidado propio y el de su hijo (1). Esta nueva etapa de vida que asume la adolescente la hace vulnerable ante la ambivalencia de consolidar su identidad personal frente a la construcción de la identidad materna.

Norma Noguera y Herly Alvarado (2) definieron a las madres adolescentes como un grupo poblacional sensible con deficiente apoyo social o familiar, bajo nivel socioeconómico y educativo, tensión emocional, sentimientos de tristeza y desesperanza, discriminación, vergüenza, baja autoestima, limitación en el proyecto de vida, fracaso en adquisición de independencia, imposibilidad de constitución de una familia estable, factores todos que afectan su calidad de vida y la del hijo y que contribuyen a elevar los índices de morbimortalidad materna-perinatal, por inasistencia y mala adherencia a los servicios de salud, al igual que por una menor disposición en el autocuidado durante proceso posparto y cuidado del recién nacido (3).

Según la Organización Mundial de la Salud (4), en el mundo cada día se producen aproximadamente 830 muertes de mujeres por causas relacionadas con el embarazo y el parto, casi todas en países en desarrollo, que en su mayoría podrían ser prevenibles. Las mujeres con mayor riesgo de presentar muerte materna son las adolescentes y mujeres mayores de 35 años. De acuerdo con lo anterior, el riesgo de muerte en jóvenes entre 15 y 19 años de edad guarda relación con trastornos hipertensivos de la gestación, hemorragias posparto, infecciones puerperales, síndrome trombo embólico, alteraciones nutricionales, anemia, depresión posparto y alteración del rol materno (5-7).

Para el 2016, la Organización Mundial de la Salud (4) detallaba que 16 millones de adolescentes en edades comprendidas entre los 15 y los 19 años dan a luz cada año y que un $95 \%$ de los nacimientos se produce en países en desarrollo. Esto representa el $11 \%$ de todos los nacimientos en el mundo. En Colombia, según datos de la Encuesta Nacional de Salud y Demografía, para diciembre del 2016 nacieron 2912 niños, hijos de madres con una edad que oscilaba entre los 10 y los 19 años, de los cuales 247 fueron atendidos en la Empresa Social del Estado (ESE) Hospital Regional de Duitama (HRD) en Boyacá (8).

El HRD, como entidad de segundo nivel, adoptó en el 2014, el programa Creciendo Juntos: Convertirse en Madre durante la Adolescencia, que promueve el cuidado integral a la gestante, pareja o familiar durante el control prenatal y que permite conocer e intervenir en factores protectores y de riesgo, que concuerdan con lo expuesto en la literatura como una problemática de salud pública (9).

Para Ramona Mercer (10), convertirse en madre es un proceso interactivo y de transición que se produce a lo largo del tiempo, en el que la madre crea un vínculo con el hijo, aprende las tareas de cuidado del rol y expresa placer y gratificación con este desde su entorno inmediato. Por su parte, el HDR, como institución amiga de la mujer y la infancia, propone el posparto como una etapa en la que enfermería puede mantener una interacción prolongada y proporcionar cuidados a la madre, hijo, pareja o familia desde su entorno inmediato, a través de la formación y el apoyo, tras el alta hospitalaria (11).

En los países en desarrollo, casi la mitad de las madres y recién nacidos no reciben cuidados profesionales durante el posparto, lo que favorece el incremento de las tasas de morbimortalidad que podrían evitarse si se aplicaran medidas sanitarias conocidas y eficaces en el parto y durante las primeras semanas tras el 
alta hospitalaria (12). Según estudios que estructuran guías de cuidado en el ámbito hospitalario y de atención primaria durante el puerperio, las intervenciones de enfermería han de ir encaminadas a prevenir la hemorragia, la infección y la enfermedad tromboembólica; a asegurar el bienestar y la comodidad de la madre; a proporcionar alivio a molestias producidas por la episiotomía o desgarros, incisión de la cesárea, hemorroides, entuertos, ingurgitación mamaria y cansancio muscular; a la vez que a facilitar la información suficiente para su autocuidado y cuidado del recién nacido (13).

Abou-Dakn et al. (14) observaron que las mujeres presentan ansiedad y temores alrededor de la crianza temprana y su papel cambiante; también falta de confianza en sí mismas como madres y su capacidad para cuidar de sí y su hijo sin apoyo profesional, lo que guarda relación con la escasa información que tiene frente a los signos y síntomas que debe vigilar y los cuidados que debe practicar para la recuperación posparto, dirigidos a prevenir, reconocer y actuar oportunamente sobre factores que generan complicaciones en la díada. A esto se suma que durante esta etapa disminuye el contacto establecido por los profesionales de la salud, el acompañamiento y la planificación del cuidado tras el alta hospitalaria, sin considerar conocimientos, experiencias, tabúes, creencias, hábitos, estilo de vida y prácticas culturales que son el resultado de la vida familiar (15).

Onaidy Bohórquez Gamba et al. (16), en un estudio de seguimiento de enfermería a la madre y al recién nacido durante el puerperio, concluyeron que madres y familiares que cuentan con escasos conocimientos y enfrentan solos el proceso de crianza del hijo y la recuperación posparto de la mamá, paralelos al proceso de asumir el rol materno, cursan con alteraciones como infección puerperal, hiperbilirrubinemia neonatal, dificultad en el establecimiento de la lactancia materna, en la madre y en el hijo. Es por esta razón por la que la educación, el acompañamiento, la resolución de dudas, la asesoría, la consulta de enfermería, la utilización de material didáctico y la inducción a la consulta de urgencias temprana son herramientas que pueden prevenir complicaciones, al minimizar los riesgos de enfermar y morir.

La responsabilidad que tienen los profesionales de enfermería radica en el compromiso continuo de brindar cuidado de calidad y calidez. En la mayor parte de las instituciones prestadoras de servicios de salud, los profesionales de enfermería garantizan el desarrollo y cumplimiento de la Estrategia Amiga de la Mujer y la Infancia (IAMI), con miras a reducir la mortalidad materna y perinatal. Para la ESE HRD, la estrategia de cuidado de enfermería promueve el cuidado integral de la madre adolescente, hijo, pareja o acompañante para la activación del rol materno durante el posparto, y favorece el cumplimiento de la estrategia IAMI en sus pasos 5, 9 y 10, que guardan relación con la educación y la atención en el puerperio al binomio madrehijo. Desde la continuidad del cuidado, apoyo y acompañamiento, garantiza entornos protectores y espacios amigables, que contribuyen que el adolescente reconfigure nuevas identidades como madre y padre desde la construcción de un proyecto de vida con significado lo largo de su vida $(17,18)$.

El objetivo del artículo es presentar la experiencia del desarrollo de una estrategia de enfermería que promueve el cuidado integral de la madre adolescente, hijo, pareja o acompañante para la activación del rol materno durante el posparto, como parte del programa Creciendo Juntos de la ESE HRD (Boyacá, Colombia).

\section{Método}

\section{Metodología para el diseño de la estrategia}

La estrategia de cuidado se diseñó aplicando la teoría convertirse en madre de Ramona Mercer, desde el sistema conceptual teórico-empírico y el modelo del marco lógico, como una herramienta para proyectos centrados en objetivos. Se tomó como centro la noción de apoyo social desde sus cuatro componentes: emocional, físico, informativo y de valoración $(11,19,20)$ como estructura dominante del programa Creciendo Juntos. 
La estrategia de cuidado se ejecutó durante cuatro meses (julio a noviembre del 2017), con un grupo de 25 madres adolescentes que fueron captadas tras su parto en el HRD y con quienes se abordaron intervenciones dentro de los 54 días siguientes. Se les informó el objetivo de la estrategia y sus beneficios, a fin de permitirles de forma autónoma, voluntaria y confidencial su participación, como parte de la responsabilidad deontológica de enfermería (21). La estrategia se estableció a partir de talleres, formato de valoración, guía de seguimiento telefónico y ayudas didácticas que contaron con una validación de contenido realizada por tres expertos del equipo de ginecobstetricia del ESE HRD y por un grupo de 10 madres adolescentes.

Entre las ayudas se encuentra el disco Girando, girando y en mi posparto nos vamos cuidando, el cual contiene signos de alarma del recién nacido y la madre; la simulación Situación de cuidado: Visualizando mi futuro, retomando mi proyecto de vida, y el apoyo de la cartografía social, como una metodología que permitió conocer la realidad de las madres adolescentes frente a la activación del rol materno. La intervención obtuvo aprobación por parte del Comité de Ética de la institución. Todo el proceso fue desarrollado y realizado por la investigadora principal y dos estudiantes de internado de Enfermería asignadas a la institución e integrantes del grupo de investigación, quienes elaboraron y aplicaron la estrategia durante el periodo propuesto.

\section{Resultados de la aplicación de la estrategia de cuidado}

Como características sociodemográficas se encontró que la edad promedio fue 17 años para el total de las 25 madres participantes. Para el 70 \% era la primera gestación. Las madres egresaron de la institución con diagnóstico de posparto normal por vía vaginal en un $70 \%$, y por cesárea, el $30 \%$, relacionada con estado insatisfactorio del feto. En cuanto al estado civil, el $60 \%$ refería vivir en unión libre junto a su pareja y la familia de alguno de los dos jóvenes. El $80 \%$ eran bachilleres, y de ellas el $40 \%$ cursaba estudios técnicos, los cuales suspendieron por el nacimiento de su hijo. El 90 \% procedía del sector urbano de la ciudad de Duitama. Del total de la población, solo 11 adolescentes contaron con la compañía de su pareja o acompañante durante el desarrollo de la estrategia de cuidado.

Los resultados se presentan desde el sistema conceptual teórico-empírico en dos momentos: primero, el diseño de la estrategia de cuidado de enfermería durante el posparto y, segundo, los indicadores empíricos que dan respuesta a su ejecución. La figura 1 esquematiza el desarrollo de la estrategia.

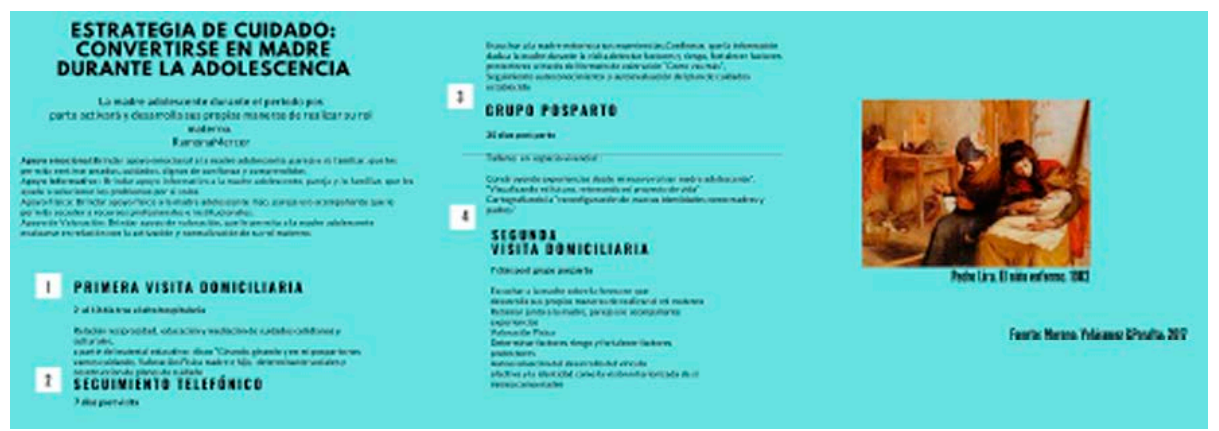

FIGURA 1.

Estrategia de cuidado: convertirse en madre durante la adolescencia, activación del rol materno durante el posparto

Fuente: elaboración propia, 2017

Indicadores empiricos: se obtuvieron a partir de la aplicación de un pre y postest, así como con el uso de la Escala de Edinburgh, la lista de chequeo para seguimiento telefónico y los formatos de valoración física para la madre y su hijo durante del posparto de la Escuela de Enfermería de la Universidad Pedagógica y Tecnológica de Colombia.

Visita posparto, apoyándote desde el hogar: en el pretest, el grupo de madres adolescentes refirió no haber recibido visita domiciliaria después del alta hospitalaria. De igual forma, las adolescentes que son madres por segunda vez refieren "no haber tenido visita durante su primer posparto". Sin embargo, los resultados del 
postest evidenciaron que el $100 \%$ de la población recibió una primera visita tras el egreso del hospital. Desde el apoyo emocional, el 80 \% de las madres adolescentes indicó que su embarazo no fue planeado ni deseado; pero sí fue aceptado. El $40 \%$ de las madres tenía buenas redes de respaldo y contaba con su familia y pareja. El 40 \% restante solo indicó el apoyo de su familia, y el $20 \%$ de las adolescentes, aunque vivía con su pareja, indicaron solo recibir sustento económico por parte de esta y sentir que la responsabilidad de crianza, cuidado y educación de los hijos, así como las labores del hogar, recaían sobre ellas.

La valoración física realizada en la visita permitió conocer el riesgo psicosocial. Todas las participantes obtuvieron resultados entre 0 y 4 puntos, lo cual indica una baja probabilidad para presentar depresión posparto. Los hallazgos relevantes durante la valoración física guardan relación con mamas congestivas secretantes, pezón y areola lacerada relacionada con la técnica de amamantamiento. El 50 \% de las madres cursa con estreñimiento y dolor asociado al desagarro o episiorrafia. Se encontró que las 25 madres adolescentes usaban un método de planificación y que de estas, un $40 \%$ refirió estar insatisfechas, ya que deseaban uno diferente.

Durante la valoración del recién nacido, el $90 \%$ presentó medidas antropométricas adecuadas respecto a peso y talla en relación con la edad gestacional. El $10 \%$ restante nació con bajo peso y fue inscrito en el Programa Canguro, con el cual hubo ganancia adecuada de peso, valorado por los servicios de pediatría y enfermería. En la primera visita, el $20 \%$ de los recién nacidos presentó tinte ictérico, asociado a incompatibilidad de grupo; otro $20 \%$ presentó ictericia neonatal patológica, diagnosticada tras remitir a los bebés a la institución de salud, donde estuvieron hospitalizados hasta obtener niveles de bilirrubinas central en límites normales. Desde el HRD, en el servicio de pediatría, se continuó con el apoyo a la madre hasta el alta del recién nacido.

En el posparto mediato se encontró que el $80 \%$ de los recién nacidos tenía lactancia materna exclusiva a libre demanda con tiempos de succión de entre 20 y 90 minutos, y el $20 \%$ restante, alimentación mixta con uso de sucedáneo, donde uno de los casos fue formulado por el pediatra del hospital, por bajo peso del recién nacido. Otro caso no contaba con fórmula y era suministrada por la madre, quien refería sentir que el bebé quedaba con hambre por su escasa secreción láctea, motivo por el cual se brindó educación y sensibilización en lactancia materna, al permitir la expresión del sentir de la adolescente.

De acuerdo con los hallazgos encontrados, en cada díada madre-hijo y su pareja o familiar se individualizó la educación y el cuidado que se iba a brindar como apoyo informativo. Se entregó el disco como material educativo a la madre.

Durante la segunda visita domiciliaria, se realizó una valoración física general y seguimiento al desarrollo del rol materno desde las herramientas proporcionadas tras la intervención. La visita se centró en un diálogo con la madre y, en algunos casos, con el padre del recién nacido. Se indagó sobre la aparición de signos de alarma, cuidados del recién nacido y de la madre, asistencia a controles, registro civil, reclamo de la hormona estimulante de la tiroides (TSH), asistencia al primer control de crecimiento y desarrollo, problemas surgidos, así como dudas y preguntas en torno al restablecimiento de procesos de autocuidado dentro de las actividades de su vida cotidiana.

Las enfermeras llevaron a cabo una valoración y establecieron la forma en que se da la activación y normalización del rol materno, y de acuerdo con esto se dieron recomendaciones, sugerencias, educación y apoyo por parte del equipo interdisciplinario, desde donde se estructuraron y consolidaron planes de cuidado para la adolescente durante el periodo posparto.

Seguimiento telefónico "Cómo vas, mamá": el grupo de madres adolescentes refirió que, tras el alta hospitalaria, no había recibido seguimiento telefónico durante el posparto; sin embargo, en la fase de consecución, práctica y recuperación física, se realizó la llamada telefónica, implementada desde la estrategia de cuidado a la adolescente en gestación Creciendo Juntos, que ejecutó el grupo de internas de enfermería en el 2014. El postest evidenció que el 100 \% de la población recibió llamadas de seguimiento. La tabla 1 resume los resultados obtenidos en el seguimiento. 
TABLA 1.

Hallazgos del seguimiento telefónico “Cómo vas, mamá”

\begin{tabular}{|c|c|c|}
\hline Indicador empírico & Hallazgos & Intervenciones \\
\hline \multirow[t]{3}{*}{$\begin{array}{l}\text { ¿Ha presentado algún signo } \\
\text { de alarma? }\end{array}$} & $\begin{array}{l}\text { Sí: } 2 \text { madres } \\
\text { ¿Cuáless?: } \\
\text { "Abundante sangrado" }\end{array}$ & $\begin{array}{l}\text { Se evalúa el sangrado en cantidad en relación con el uso de toalla } \\
\text { higiénica. Se informa el riesgo. Se remite a la institución donde se hace } \\
\text { seguimiento, toman exámenes paraclínicos y valoran. }\end{array}$ \\
\hline & "Dolor bajito" & $\begin{array}{l}\text { Se evalúan síntomas asociados, descartando alteración en la cavidad } \\
\text { uterina y de origen genitourinario. Se dan indicaciones frente a la } \\
\text { presencia de signos de infección. }\end{array}$ \\
\hline & No: 23 madres & $\begin{array}{l}\text { Se retoma la información del material educativo relacionado con signos de } \\
\text { alarma. }\end{array}$ \\
\hline \multirow{2}{*}{$\begin{array}{l}\text { ¿Cómo se siente } \\
\text { emocionalmente? }\end{array}$} & Bien: 22 madres & Fortalecer factores protectores \\
\hline & Mal: 3 madres. "Sentí tristeza la primera semana" & $\begin{array}{l}\text { Se informa y se remite para valoración y seguimiento por trabajo } \\
\text { social/psicología. }\end{array}$ \\
\hline \multirow{3}{*}{$\begin{array}{l}\text { ¿Cómo se ha sentido con el } \\
\text { método de planificación? }\end{array}$} & Bien: 20 madres & \multirow{3}{*}{$\begin{array}{l}\text { Sc brinda educación sobre el mecanismo de acción del implante } \\
\text { subdérmico; de igual modo, se informa de posibles efectos secundarios. } \\
\text { Dado que la respuesta de la madre se relaciona con una deficiente asesoría } \\
\text { y consejería en métodos de planificación, se remite a consulta para que, de } \\
\text { acuerdo con su individualidad y criterios de elegibilidad, pueda acceder al } \\
\text { método más adecuado. }\end{array}$} \\
\hline & $\begin{array}{l}\text { Mal: } 5 \text { madres } \\
\text { "Me dio dolor de cabeza y mal genio en las dos } \\
\text { primeras semanas" (implante subdérmico) }\end{array}$ & \\
\hline & $\begin{array}{l}\text { "Quiero planificar con el implante subdérmico" } \\
\text { (inyectable trimestral) }\end{array}$ & \\
\hline \multirow[t]{5}{*}{$\begin{array}{l}\text { ¿Su hijo ha presentado } \\
\text { algún signo de alarma? }\end{array}$} & $\begin{array}{l}\text { Si: } 3 \text { madres } \\
\text { "No hacia deposición desde hace } 3 \text { días" }\end{array}$ & \multirow[t]{2}{*}{$\begin{array}{l}\text { Se indaga si acudió al centro de salud y se encontró que solo una de las } \\
\text { madres asiste a la institución con su recién nacido, donde le indican que el } \\
\text { estreńimiento es normal, y refiere que al día siguiente presenta deposición. }\end{array}$} \\
\hline & "Fiebre" & \\
\hline & "Congestión nasal" & $\begin{array}{l}\text { Se brinda educación en el patrón intestinal que irá adquiriendo el recién } \\
\text { nacido y signo de alarma de estreñimiento. }\end{array}$ \\
\hline & & $\begin{array}{l}\text { El recién nacido que presenta fiebre fue medicado por la madre con } \\
\text { acetaminofén; se brinda educación en efectos adversos y se sensibiliza a } \\
\text { acudir a la institución de salud frente algún signo de alarma que presente } \\
\text { el hijo. }\end{array}$ \\
\hline & No: 19 madres & $\begin{array}{l}\text { Con las madres que sus hijos no presentaron signos de alarma se refuerzan } \\
\text { cada uno de estos. }\end{array}$ \\
\hline \multirow[t]{4}{*}{$\begin{array}{l}\text { ¿Qué cuidados está } \\
\text { teniendo con su bebé? }\end{array}$} & $\begin{array}{l}\text { Terapia de sol, bańo diario, lactancia materna, } \\
\text { cuidados cordón umbilical (24 madres) }\end{array}$ & $\begin{array}{l}\text { Se brinda educación sobre la importancia de realizar caricias, masajes y } \\
\text { hablar al recién nacido como medio de estimulación y fortalecimiento del } \\
\text { vínculo afectivo. }\end{array}$ \\
\hline & $\begin{array}{l}\text { Cuidados con el plan canguro al recién nacido } \\
\text { por bajo peso ( } 1 \text { madre) }\end{array}$ & $\begin{array}{l}\text { Se brinda educación en la importancia del contacto piel a piel como } \\
\text { medio para mejorar la ganancia de peso, así como la lactancia materna. }\end{array}$ \\
\hline & $\begin{array}{l}\text { Toma de exámenes paraclínicos al bebé por } \\
\text { antecedente materno de estreptococo B }\end{array}$ & Se brinda educación sobre la importancia de reclamar los resultados y \\
\hline & $\begin{array}{l}\text { hemolítico positivo y toxoplasmosis materna ( } 1 \\
\text { madre) }\end{array}$ & $\begin{array}{l}\text { asistrir con estos a control por pediatría, para seguimiento del recién } \\
\text { nacido. }\end{array}$ \\
\hline \multirow{3}{*}{$\begin{array}{l}\text { ¿Con qué está alimentando } \\
\text { a su bebé? }\end{array}$} & Lactancia materna exclusiva: 23 madres & \multirow{2}{*}{$\begin{array}{l}\text { Se refuerza educación sobre importancia, beneficios y técnica de la } \\
\text { lactancia materna. }\end{array}$} \\
\hline & Lactancia materna más sucedáneo: 2 madres & \\
\hline & & $\begin{array}{l}\text { Usuarias refieren alimentación mixta por baja producción láctea y por } \\
\text { decisión propia. Se sugiere aumentar la frecuencia en succión para } \\
\text { aumentar producción. }\end{array}$ \\
\hline \multirow{2}{*}{$\begin{array}{l}\text { ¿Reclamo el resultado del } \\
\text { TSH neonatal? }\end{array}$} & Si: 24 madres & \multirow{2}{*}{$\begin{array}{l}\text { Se direcciona al laboratorio para reclamar el resultado del TSH neonatal y } \\
\text { realizar inscripción para control de crecimiento y desarrollo. }\end{array}$} \\
\hline & No: 1 madre & \\
\hline
\end{tabular}

Fuente: elaboración propia, 2017

Grupo posparto: se realizó en el HRD y asistieron las 25 madres adolescentes, junto a sus hijos y once acompañantes, lo cual representó una limitante para el desarrollo de la propuesta. Desde el apoyo emocional e informativo, las madres adolescentes refieren que durante el posparto no se les había permitido expresar sus sentimientos y conocer las experiencias de otras jóvenes, ya que solo les realizaban la consulta, a diferencia de lo manifestado tras la intervención de cuidado, cuando todas expresaron sus sentimientos y compartieron las experiencias con otras adolescentes y asistieron a talleres grupales donde se les brindó información que ha sido útil dentro de su rol materno.

La cartografía permitió documentar e interpretar la situación de las madres a partir de la construcción de criterios y correlación de experiencias (22). La figura 2 muestra el territorio existencial, representado por la silueta de una madre y su hijo como símbolo de vínculo afectivo. La naturaleza icónica de la imagen representa desde el nombre que otorgan las adolescentes hasta la percepción frente a su rol materno. 


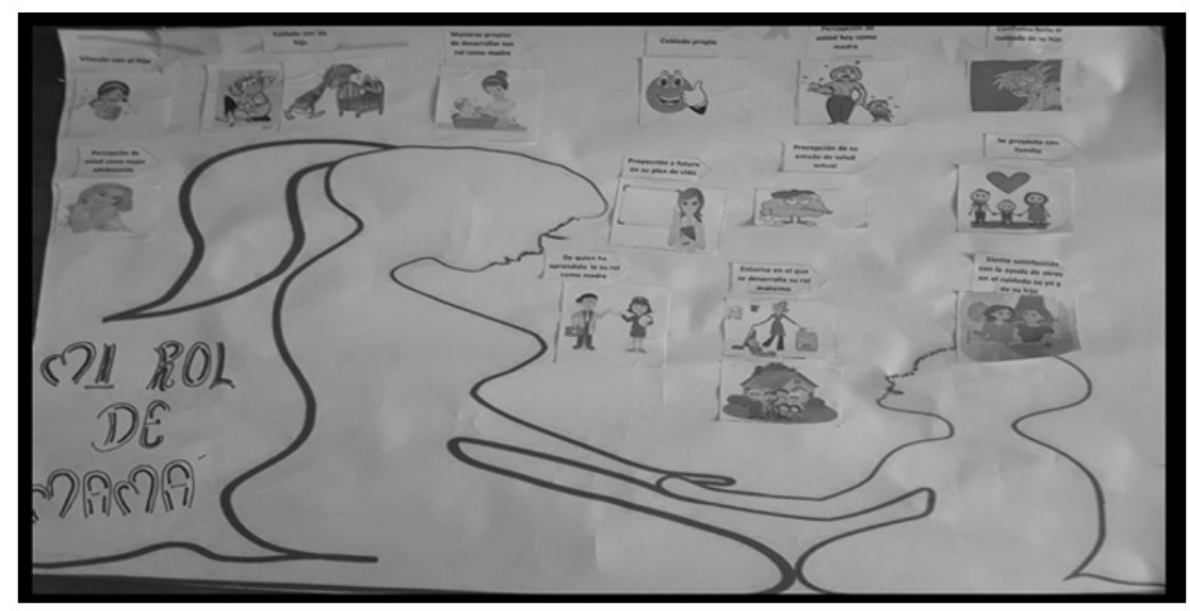

FIGURA 2.

Mapa colectivo: cartografía mi rol de mamá. Adolescentes del grupo posparto (Duitama, Boyacá, Colombia, 2017)

Fuente: elaboración propia, 2017

Desde el contenido del mapa, el indicador "El cuidado con el hijo" muestra dos imágenes: la primera, una joven que logra alimentar a su hijo y, otra, una madre cansada que atiende al llanto del recién nacido, lo cual es descrito por el grupo como "el cuidado a nuestro hijo nos da como cosa de que jay! que meta aquí que coja que haga, da como miedo" y "a veces nos sentimos cansadas y cuando se ponen a llorar es cuando más nos estresamos". Desde el indicador de madre adolescente se observa una joven alegre que es descrita como "nos va bien y felices".

El indicador "Proyección a futuro" muestra la imagen de una joven que carga un libro en sus manos, lo cual indica para el grupo que "nuestro plan de vida es seguir estudiando, nos vemos siendo unas grandes... Digamos lo que cada una quiere ser... Como contabilidad". El indicador "Familia", lo representan con la imagen de una familia de la cual expresan "nos proyectamos conformado una familia, siendo felices y estando enamoradas". El indicador "Percepción de su estado de salud" es representado por un cerebro enfermo que a la socialización describen como "nos sentimos agotadas".

El indicador "Entorno", en el que se desarrolla su rol como madre, es representado por una mujer que limpia, lo que para el grupo es "nuestro entorno es la casa, donde como madre es pues... Hacer aseo y seguir pendientes del bebé y la familia”; "pero sabemos que volveremos a retomar nuestras vidas, a estudiar y seguir". El indicador "De quien ha aprendido su rol como madre" lo representan con la imagen del personal de la salud que a la socialización identifica "las personas que tienen más experiencia, ustedes y nuestros padres". Lo que es coherente con el indicador "Satisfacción" con la ayuda de otros en el cuidado de su yo y de su hijo, donde se encuentra que: "Sí nos gusta que nos digan cosas que no sabemos... Pero también que nos dejen hacer las cosas que ya nos salen bien".

\section{Discusión}

La estrategia de cuidado de enfermería desarrollada evidencia el proceso continuo de convertirse en madre, durante la fase de consecución, práctica y recuperación física (primeras dos semanas posparto) y normalización (de la segunda semana posparto hasta los cuatro meses), las cuales comprenden el periodo en el que la madre activa y desarrolla sus propias maneras de realizar el rol, y hace que se adapte a su estilo de vida, basándose en experiencias pasadas y en objetivos futuros, al influir en el rol maternal en desarrollo y en el niño (23). 
Por otra parte, el concepto de apoyo social hace referencia a "la cantidad de ayuda que recibe realmente la madre, la satisfacción que produce esa ayuda y los profesionales de enfermería que la proporcionan" (24), el cual se desarrolló por medio de la visita domiciliaria, el seguimiento telefónico y el grupo posparto, como intervenciones que permitieron brindar cuidado individual y grupal, personalizado y de reciprocidad a la mujer, al recién nacido y a su pareja o acompañante.

Los resultados expuestos desde la percepción de las adolescentes son coherentes con lo expuesto por Mercer, en los cuales la autoestima y la confianza son importantes para iniciar la fase de normalización, pues es ahí cuando la madre empieza a desarrollar maneras propias de realizar el rol, basándose en experiencias y enseñanzas del sistema social. Por ello, es importante la comunicación y la identificación de necesidades, para mejorar la atención y promover las actitudes de crianza en el tiempo (25).

La fase de activación y normalización del rol materno hace parte del estilo de vida de la adolescente, quien desde su vivencia lo expone como una visión interiorizada de sí misma como mujer y madre. Es el momento en el cual el hijo, su padre, el entorno social y cultural serán determinantes en la consolidación de la identidad materna, como aquel proceso que se desarrollará de manera efectiva a lo largo del tiempo, y que iniciará su consolidación tras el finalizar esta fase, al dar apertura a la fase de integración, cuando la madre adolescente logra aprender las tareas para trascender hacia la activación y normalización del rol materno: convertirse en madre, para lo cual no estaba preparada (25). Sin embargo, la dificultad de no contar en las intervenciones con la presencia del padre es un asunto que culturalmente puede incidir en el logro y consecución de las etapas de adaptación del rol; por lo general, son las madres, hermanas o amigas quienes acompañan a la adolescente en las diferentes actividades.

Para las madres adolescentes, el acompañamiento mediante el desarrollo de las estrategias de cuidado lideradas por enfermería, con acompañamiento de la pareja, permite activar el rol, mejorar los vínculos, al igual que manejar emociones, ansiedad y depresión (26). Las madres serán capaces de continuar con un proyecto de vida en el cual confluyen el mantenimiento de su calidad de vida y el de su hijo.

\section{Conclusiones}

La experiencia permitió desarrollar una estrategia de enfermería que promovió el cuidado integral de la madre adolescente, al proporcionarle herramientas desde el apoyo social (emocional, informativo, físico y de valoración) y permitirle a la adolescente la reconstrucción de su percepción como mujer y madre desde la autoaceptación, la autoestima, la coherencia o la discrepancia entre la identidad actual y la futura. Para Mercer es un eslabón en la interiorización del convertirse en madre, desde la visualización de nuevo proyecto de vida.

La estrategia valida la visibilidad del cuidado, sustentado en una teoría de enfermería, al dar respuesta a las necesidades humanas; así como la afirmación de conceptos utilizados en su diseño, donde el convertirse en madre no se activa sin la influencia del cuidado estructurado brindado por enfermería, que lidera el equipo de salud y permite a las madres, pareja o familiar disminuir sus miedos, conocer y vivir el periodo posparto.

\section{Agradecimientos}

Al grupo de investigación Gestión y Calidad en Enfermería (Escuela de Enfermería) de la Universidad Pedagógica y Tecnológica de Colombia y al Hospital Regional de Duitama. 


\section{Referencias}

1. Vélez E, Figueredo L. Impacto psicosocial del embarazo en las adolescentes. Rev Educ Valores [internet]. 2015;1(23):18-28. Disponible en: http://servicio.bc.uc.edu.ve/multidisciplinarias/educacion-en-valores/v1n2 3/art03.pdf

2. Noguera N, Alvarado H. Embarazo en adolescentes: una mirada desde el cuidado de enfermería. Rev Colomb Enferm [internet]. 2012;7(1):151-60. Disponible en: https://dialnet.unirioja.es/servlet/articulo?codigo $=4069201$

3. Mora Cancino A, Hernández Valencia M. Embarazo en la adolescencia. Ginecol Obstet Mex [internet]. 2015;83:294-301. Disponible en: https://www.medigraphic.com/pdfs/ginobsmex/gom-2015/gom155e.pdf

4. Organización Mundial de la Salud. Mortalidad materna [internet]. 2016. Disponible en: http://www.who.int/me diacentre/factsheets/fs348/es/

5. Cox J, Holden J, Sagovsky R. Escala de Depresión de Post-Parto de Edinburgh EPDS. Br J Psychat. 1987;150:1-2.

6. Henao L, Cañón M, Herrera M. Caracterización de la morbilidad materna extrema en la clínica universitaria Colombia: una serie de casos. Rev Médica Sanitas [internet]. 2016;19(2):66-77. Disponible en: http://www.unisanitas.edu.co/Revista/59/CARACTERIZACION_DE_LA_MORBILIDAD_MATER NA_EXTREMA_EN_LA_CLINICA_UNIVERSITARIA_COLOMBIA_UNA_SERIE_DE_CASOS.pdf

7. Colmenares Z, Montero L, Reina R, González Z. Intervención de enfermería durante la clínica prenatal y conocimiento sobre riesgos fisiológicos de la adolescente embarazada. Rev Enferm Global [internet]. 2010;18:1-10. Disponible en: http://scielo.isciii.es/scielo.php?script=sci_arttext\&pid=S1695-614120100001 00005\&lng=es

8. Ministerio de Salud y Protección Social. Encuesta Nacional de Demografía y Salud (ENDS). Tomo I [internet]. Bogotá; 2015. Disponible en: https://profamilia.org.co/wp-content/uploads/2018/12/ENDS-TOMO-I.pdf

9. Binstock, Georgina, Näslund-Hadley E. Maternidad adolescente y su impacto sobre las trayectorias educativas y laborales de mujeres de sectores populares urbanos de Paraguay. Papeles de Población. 2013;19(78):15-40.

10. Mercer R. Becoming a mother versus maternal role attainment. J Nurs Scholarsh [internet]. 2004;36(3):226-32. Disponible en: https://www.ncbi.nlm.nih.gov/pubmed/15495491

11. Alvarado L, Guarín L, Cañón W. Adopción del rol maternal de la teorista Ramona Mercer al cuidado de enfermería binomio madre-hijo: reporte de caso en la unidad materno infantil. Rev Cuidarte. 2011;2(2):195-201. https:// doi.org/10.15649/cuidarte.v2i1.57

12. Parada Rico D. Adopción de prácticas saludables en puérperas adolescentes. Rev Cuidarte. 2013;4(1):450-8.http s://doi.org/10.15649/cuidarte.v4i1.4

13. Belfort D, Clark S, Saade G, et al. Reinternaciones posparto en el periodo puerperal. J Obstet Gynecol. 2010;202(35):1-7. http://dx.doi.org/10.1016/j.ajog.2009.08.029

14. Abou-Dakn M, Richardt A, Schaefer-Graf U, Wöckel A. Inflammatory breast diseases during lactation: milk stasis, puerperal mastitis, abscesses of the breast, and malignant tumors - current and evidence-based strategies for diagnosis and therapy. Rev Breast Care. 2010;5:33-37. https://doi.org/10.1159/000272223

15. Laza C, Puerto M. Cuidados genéricos para restablecer el equilibrio durante el puerperio. Rev Cubana Enferm [internet]. 2011;27(1):88-97. Disponible en: https://www.researchgate.net/publication/317522092_Cuidado s_genericos_para_restablecer_el_equilibrio_durante_el_puerperio

16. Bohórquez Gamba OA, Rosas Santana A, Pérez LJ, Munévar RY. Seguimiento de enfermería a la madre y al recién nacido durante el puerperio: traspasando las barreras hospitalarias. Av Enferm [internet]. 2009;27(2). Disponible en: https://revistas.unal.edu.co/index.php/avenferm/article/view/12976

17. Alvarado R, Martínez O, Baquero M, Valencia C, Ruiz Á. Problemática en torno al embarazo en adolescentes de 14 a 19 años, en Nocaima (Cundinamarca). Cienc Salud [internet]. 2007;5(1):40-52. Disponible en: https://r evistas.urosario.edu.co/index.php/revsalud/article/view/497

18. Navarro C, Navarrete L, Lara M. Factores asociados a la percepción de eficacia materna durante el posparto. Salud Ment [internet]. 2011;34(1):37-43. Disponible en: http://www.scielo.org.mx/scielo.php? script $=$ sci_arttext\&pid $=$ S0185-33252011000100005\&lng=es\&nrm=iso $>$. ISSN 0185-3325 
19. Quintero Rondón A, Rojas Betancur H. El embarazo a temprana edad, un análisis desde la perspectiva de madres adolescentes. Rev Virtual Univ Catól Norte [internet]. 2015;44:222-37.Disponible en: https://www.redalyc.o $\mathrm{rg} /$ articulo.oa?id=1942/194238608016

20. Congreso de Colombia. Deontológica para el ejercicio de la profesión de Enfermería en Colombia. Ley 911 del 2004. Diario Oficial 45.693 de 6 de octubre de 2004 [internet]. 2004. Disponible en: http://www.mineducaci on.gov.co/1759/articles-105034_archivo_pdf.pdf

21. Barragán D, Amador JC. La cartografía social-pedagógica: una oportunidad para producir conocimiento y repensarla educación. Itinerario Educativo. 2014;28(64):127-41. https://doi.org/10.21500/01212753.1422

22. Borges A, Padula M, Schenker M. Instrumento para a atenção integral: do pré-natal ao puerpério. Rev Bras Med Fam Comunidade [internet]. 2008;4(13):53-60. Disponible en: http://www.rbmfc.org.br/index.php/rbmfc/a rticle/view/219

23. Berlanga S, Pérez R, Vizcaya M, Tarrés R. Apoyo social formal a un grupo de madres con hijos Menores de un año. Aquichan [internet]. 2013;13(2):206-15. Disponible en: https://aquichan.unisabana.edu.co/index.php/aquic han/article/view/2089/3224

24. Acosta Romo MF, Cabrera Bravo N, Basante Castro Y, Jurado D. Sentimientos que experimentan los padres en el difícil camino de la hospitalización de sus hijos prematuros: un aporte al cuidado humanizado. Rev Univ Salud. 2017;19(1):17-25.

25. Espinoza E. La madre, seguridad y visión de futuro frente al rol materno. Rev Peru Epidemiol [internet]. 2011;15(2):102-5. Disponible en: http://www.revenfermeria.sld.cu/index.php/enf/article/view/815

26. Ramírez Peláez H, Rodríguez Gallego I. Beneficios del acompañamiento a la mujer por parte de su pareja durante el embarazo, el parto y el puerperio en relación con el vínculo paternofilial: revisión bibliográfica. Matronas Profesión [internet]. 2014; 15(4):1-6. Disponible en: https://www.federacion-matronas.org/wp-content/uplo ads/2018/01/revision-beneficios-pareja.pdf

\section{Notas}

* Artículo de reflexión

Limitaciones: El número de madres adolescentes que cumplieran criterios de participación como parto institucional eutócico, adolescentes entre 15 y 19 años, recién nacido vivo sin criterios de unidad de cuidados intensivos y lugar de residencia. De igual manera, la vinculación, la pareja o familiar, durante el desarrollo del grupo posparto, debido a la disponibilidad de horarios y las actividades laborales.

Conflicto de intereses: las autoras declaran no tener conflictos de interés.

\section{Licencia Creative Commons CC BY 4.0}

Cómo citar este artículo: Moreno CM, Peralta Cruz A, Velásquez Monroy N. Convertirse en madre durante la adolescencia: transiciones en el rol materno. Investig Enferm Imagen Desarr. 2020;22. https://doi.org/1 0.11144.Javeriana.ie22.cmda 